\title{
Accurate Time Delay Estimation based
}

\section{Passive Localization}

\author{
Kenneth W. K. Lui, Frankie K. W. Chan and H. C. So \\ Department of Electronic Engineering \\ City University of Hong Kong, Tat Chee Avenue, Kowloon, Hong Kong \\ Email : 50469990@student.cityu.edu.hk,ckwf@hkexperts.com, hcso@ee.cityu.edu.hk
}

\begin{abstract}
A conventional approach for source localization is to utilize time delay measurements of the emitted signal received at an array of sensors. The time delay information is then employed to construct a set of hyperbolic equations from which the target position can be determined. In this paper, we utilize semidefinite programming (SDP) technique to derive a passive source localization algorithm which can integrate the available a priori knowledge such as admissible target range and other cues. It is shown that the SDP method is superior to the well-known two-step weighted least squares method at lower signal-to-noise ratio conditions.
\end{abstract}

\section{Index Terms}

Time delay estimation, source localization, semi-definite programming

\section{INTRODUCTION}

Passive source localization has been an important research topic in signal processing because of its many applications such as search-and-rescue [1], speaker tracking [2], mobile phone location [3] and navigation [4]. Time delay estimation method [2]-[3] is a popular positioning strategy and it proceeds in a two-step fashion. First, a set of time-difference-of-arrival (TDOA) measurements of the passive signal received at spatially separated receivers is obtained. In the second step, we multiply the measured time 
delays by the known signal propagation speed to give the range difference information for constructing a set of hyperbolic equations that are highly nonlinear, from which the source position can be determined with the knowledge of the sensor array geometry.

An optimum approach for determining the source location is to solve the hyperbolic equations using nonlinear least squares (NLS) [5] where Taylor-series expansion is utilized for linearization and the solution is obtained in an iterative manner. However, the NLS scheme cannot guarantee global convergence unless the initial guess is sufficiently close to the true position. Alternatively, the nonlinear equations can be reorganized into a set of linear equations via introducing an intermediate variable, which is a function of the source position, so that a global solution can be acquired. The two-step weighted least squares (WLS) algorithm [6] is a representative example of this approach which involves two WLS operations: the first is to solve the linear equations while the second is to utilize the known relationship between the introduced variable and position coordinates. However, the two-step WLS method is able to provide optimum estimation performance only when the measurement errors are sufficiently small. In this paper, we base on the second approach to derive a semi-definite programming (SDP) [7] source positioning algorithm, which allows integration of available prior knowledge such as admissible source range and other cues. Simulation results illustrate that the proposed algorithm outperforms [6] at larger noise conditions and its estimation performance can approach Cramér-Rao lower bound (CRLB).

\section{Algorithm Development}

We first introduce the notations. Bold upper case symbols denote matrices and bold lower case symbols denote vectors. The $\mathbf{0}_{m \times n}$ is the $m \times n$ zero matrix, $\mathbf{I}_{m}$ is the $m \times m$ identity matrix, ${ }^{T}$ denotes transpose operator, ${ }^{-1}$ represents matrix inverse, $\operatorname{Tr}$ is the trace operator, vec is the vectorization operator, $\bigcap$ is the intersection operator, $\mathbb{E}$ is the expectation operator and $\|\mathbf{x}\|_{2}$ represents the 2-norm of a vector $\mathbf{x}$. For two symmetric matrices $\mathbf{A}$ and $\mathbf{B}, \mathbf{A} \succeq \mathbf{B}$ means that $\mathbf{A}-\mathbf{B}$ is positive semi-definite. While for two vectors $\mathbf{a}$ and $\mathbf{b}, \mathbf{a} \succeq \mathbf{b}$ means that the former is element-wisely greater or equal to the latter. Let $\mathbf{x}=[x, y, z]^{T}$ and $\mathbf{x}_{i}=\left[x_{i}, y_{i}, z_{i}\right]^{T}, i=1,2, \cdots, M$, be the unknown source location and known receiver positions with $M \geq 4$, respectively. A total of $M(M-1) / 2$ TDOA measurements will be obtained and they are 
then processed [8] to produce $(M-1)$ independent estimates which are relative to a particular receiver. Multiplying by the known signal propagation speed, we have $(M-1)$ range difference measurements. Without loss of generality, we let the first sensor be the reference and assume that it is closest to the target. The range difference measurements with respect to $\mathbf{x}_{1}$, denoted by $d_{i 1}$, is modeled as:

$$
d_{i 1}=d_{i 1}^{o}+n_{i 1}, \quad i=2,3, \cdots, M
$$

where $d_{i 1}^{o}=\left\|\mathbf{x}-\mathbf{x}_{i}\right\|_{2}-\left\|\mathbf{x}-\mathbf{x}_{1}\right\|_{2}>0$ is the true range difference while the measurement error $n_{i 1}$ is a zero-mean Gaussian process. The covariance of $\mathbf{n}=\left[n_{21}, n_{31}, \cdots, n_{M 1}\right]^{T}$, denoted by $\mathbf{N}=\mathbb{E}\left\{\mathbf{n n}^{T}\right\}$ is assumed known up to a scalar. The task is to find $\mathrm{x}$ using the $(M-1)$ measurements of $\left\{d_{i 1}\right\}$. Following [6], we introduce an intermediate variable, say, $r$, of the form:

$$
r=\left\|\mathbf{x}-\mathbf{x}_{1}\right\|_{2}
$$

Assuming sufficiently small noise conditions such that the terms $n_{i 1}^{2}$ and $2 n_{i 1} \mid \mathbf{x}_{i}-\mathbf{x}_{1} \|_{2}$ can be neglected, a set of linear equations is obtained by squaring both sides of (1) with the use of (2):

$$
\left(\mathbf{x}_{i}-\mathbf{x}_{1}\right)^{T} \mathbf{x}+d_{i 1} r \approx \frac{1}{2}\left(\left\|\mathbf{x}_{i}-\mathbf{x}_{1}\right\|_{2}^{2}-d_{i 1}^{2}\right), \quad i=2,3, \cdots, M
$$

Denoting $\boldsymbol{\theta}=\left[\begin{array}{ll}\mathbf{x}^{T} & r\end{array}\right]^{T}$, we express (3) in matrix form:

$$
\mathrm{G} \boldsymbol{\theta} \approx \mathbf{h}
$$

where

$$
\mathbf{G}=\left[\begin{array}{cc}
\mathbf{x}_{2}^{T}-\mathbf{x}_{1}^{T} & d_{21} \\
\mathbf{x}_{3}^{T}-\mathbf{x}_{1}^{T} & d_{31} \\
\vdots & \vdots \\
\mathbf{x}_{M}^{T}-\mathbf{x}_{1}^{T} & d_{M 1}
\end{array}\right] \quad \text { and } \quad \mathbf{h}=\frac{1}{2}\left[\begin{array}{c}
\left\|\mathbf{x}_{2}-\mathbf{x}_{1}\right\|_{2}^{2}-d_{21}^{2} \\
\left\|\mathbf{x}_{3}-\mathbf{x}_{1}\right\|_{2}^{2}-d_{31}^{2} \\
\vdots \\
\left\|\mathbf{x}_{M}-\mathbf{x}_{1}\right\|_{2}^{2}-d_{M 1}^{2}
\end{array}\right]
$$

In this work, we propose to minimize the following WLS cost function:

$$
(\mathbf{G} \boldsymbol{\theta}-\mathbf{h})^{T} \mathbf{W}(\mathbf{G} \boldsymbol{\theta}-\mathbf{h})
$$

where $\mathbf{W}$ is the optimum weighting matrix and its ideal form is [6]:

$$
\mathbf{W}=\left(\mathbf{B}^{T} \mathbf{N B}\right)^{-1}
$$


with $\mathbf{B}=\operatorname{diag}\left(d_{21}^{o}+r, d_{31}^{o}+r, \cdots, d_{M 1}^{o}+r\right)$, subject to equality and inequality constraints deduced from all available information. The first constraint is the equality of (2) while the inequality constraints are obtained by exploiting the admissible physical regions bounded by the environment and source, denoted by $\mathscr{P}$ and $\mathscr{S}$, as follows. First we consider to form a line whose endpoints are the reference sensor and the $i$ th sensor. The plane which is perpendicular to this line and contains its midpoint will divide the space into 2 regions: one includes the $i$ th sensor and the other contains the reference sensor, denoted by $\mathscr{R}_{i}$ and $\mathscr{R}_{1 i}$, respectively. Noting that the first sensor is closest to $\mathrm{x}$, a more compact region in which the source must be located, denoted by $\mathscr{R}$, is determined as $\mathscr{R}=\mathscr{R}_{12} \bigcap \mathscr{R}_{13} \bigcap \cdots \bigcap \mathscr{R}_{1 M} \bigcap \mathscr{P}$. Together with the additional cues derived from the target, namely, $\mathscr{S}$, the lower and upper limits for $\mathrm{x}$, denoted by $\mathbf{x}_{l}$ and $\mathbf{x}_{u}$, will be

$$
\begin{aligned}
& \mathbf{x}_{l}=\arg \min _{\mathbf{p} \in\{\mathscr{R} \cap \mathscr{S}\}}\{\mathbf{p}\} \\
& \mathbf{x}_{u}=\arg \max _{\mathbf{p} \in\{\mathscr{R} \cap \mathscr{S}\}}\{\mathbf{p}\}
\end{aligned}
$$

In a similar manner, the lower and upper bounds for $r$, denoted by $r_{l}$ and $r_{u}$, are determined as

$$
\begin{aligned}
& r_{l}=\min _{\mathbf{p} \in\{\mathscr{R} \cap \mathscr{S}\}}\left\{\left\|\mathbf{p}-\mathbf{x}_{1}\right\|_{2}\right\} \\
& r_{u}=\max _{\mathbf{p} \in\{\mathscr{R} \cap \mathscr{S}\}}\left\{\left\|\mathbf{p}-\mathbf{x}_{1}\right\|_{2}\right\}
\end{aligned}
$$

Up to now, our optimization problem is:

$$
\begin{aligned}
& \min _{\boldsymbol{\theta}}(\mathbf{G} \boldsymbol{\theta}-\mathbf{h})^{T} \mathbf{W}(\mathbf{G} \boldsymbol{\theta}-\mathbf{h}) \\
& \text { s.t. } \quad r=\left\|\mathbf{x}-\mathbf{x}_{1}\right\|_{2} \\
& \quad \boldsymbol{\theta}_{u} \succeq \boldsymbol{\theta} \succeq \boldsymbol{\theta}_{l}
\end{aligned}
$$

where $\boldsymbol{\theta}_{u}=\left[\mathbf{x}_{u}^{T}, r_{u}\right]^{T}$ and $\boldsymbol{\theta}_{l}=\left[\mathbf{x}_{l}^{T}, r_{l}\right]^{T}$. It is worthy to point out that [6] solves (10) in an iterative manner as $\mathbf{W}$ is a function of $\boldsymbol{\theta}$ and (11) is exploited implicitly in its second step. Our major difference is that we try to solve (10)-(11) in a more explicit manner and to further increase the estimation accuracy with the use of (12). Note that it is impractical to tackle (10)-(12) using the Lagrange multipliers because numerous variables will be introduced in the solving procedure. Instead, we approximate (10)-(12) with the use of the SDP technique as follows. First, we introduce a variable matrix $\boldsymbol{\Theta}=\boldsymbol{\theta} \boldsymbol{\theta}^{T}$ and drop the 
constant term $\mathbf{h}^{T} \mathbf{W h}$ in (6) to rewrite the objective function as

$$
\mathbf{G}^{T} \mathbf{W G} \Theta-2 \boldsymbol{\theta}^{T} \mathbf{G}^{T} \mathbf{W h}
$$

Squaring both sides of (11) and letting $\mathbf{P}=\operatorname{diag}(1,1,1,-1)$ and $\mathbf{Q}=\left[\mathbf{I}_{3}, \mathbf{0}_{3 \times 1}\right]$, the equality constraint is modified as:

$$
\begin{aligned}
& r^{2}=\left\|\mathbf{x}-\mathbf{x}_{1}\right\|_{2}^{2} \\
\Rightarrow & r^{2}=\mathbf{x}^{T} \mathbf{x}-2 \mathbf{x}_{1}^{T} \mathbf{x}+\mathbf{x}_{1}^{T} \mathbf{x}_{1} \\
\Rightarrow & \operatorname{Tr}(\mathbf{P} \boldsymbol{\Theta})+\mathbf{x}_{1}^{T} \mathbf{x}_{1}-2 \mathbf{x}_{1}^{T} \mathbf{Q} \boldsymbol{\theta}=0
\end{aligned}
$$

In order to establish relations between $\Theta$ and $\boldsymbol{\theta}$, we perform self-multiplication and cross-multiplication on (12) to generate another set of linear constraints:

$$
\begin{aligned}
& \operatorname{vec}\left(\boldsymbol{\theta}_{u} \boldsymbol{\theta}_{u}^{T}-\boldsymbol{\theta}_{u} \boldsymbol{\theta}-\boldsymbol{\theta} \boldsymbol{\theta}_{u}^{T}\right) \succeq \operatorname{vec}(-\boldsymbol{\Theta}) \\
& \operatorname{vec}\left(-\boldsymbol{\theta}_{l} \boldsymbol{\theta}^{T}-\boldsymbol{\theta} \boldsymbol{\theta}_{l}^{T}+\boldsymbol{\theta}_{l} \boldsymbol{\theta}_{l}^{T}\right) \succeq \operatorname{vec}(-\boldsymbol{\Theta}) \\
& \operatorname{vec}\left(\boldsymbol{\theta}_{u} \boldsymbol{\theta}^{T}-\boldsymbol{\theta}_{u} \boldsymbol{\theta}_{l}^{T}+\boldsymbol{\theta} \boldsymbol{\theta}_{l}^{T}\right) \succeq \operatorname{vec}(\boldsymbol{\Theta})
\end{aligned}
$$

By relaxing $\boldsymbol{\Theta}=\boldsymbol{\theta} \boldsymbol{\theta}^{T}$ as $\boldsymbol{\Theta} \succeq \boldsymbol{\theta} \boldsymbol{\theta}^{T}$ [7], our proposed SDP algorithm is finalized as

$$
\begin{array}{ll} 
& \min _{\boldsymbol{\theta}, \boldsymbol{\Theta}} \mathbf{G}^{T} \mathbf{W G} \boldsymbol{\Theta}-2 \boldsymbol{\theta}^{T} \mathbf{G}^{T} \mathbf{W h} \\
\text { s.t. } \quad & \operatorname{Tr}(\mathbf{P} \boldsymbol{\Theta})+\mathbf{x}_{1}^{T} \mathbf{x}_{1}-2 \mathbf{x}_{1}^{T} \mathbf{Q} \boldsymbol{\theta}=0 \\
& {\left[\begin{array}{rr}
\boldsymbol{\Theta} & \boldsymbol{\theta} \\
\boldsymbol{\theta}^{T} & 1
\end{array}\right] \succeq \mathbf{0}_{5 \times 5}} \\
& \operatorname{vec}\left(\boldsymbol{\theta}_{u} \boldsymbol{\theta}_{u}^{T}-\boldsymbol{\theta}_{u} \boldsymbol{\theta}-\boldsymbol{\theta} \boldsymbol{\theta}_{u}^{T}\right) \succeq \operatorname{vec}(-\boldsymbol{\Theta}) \\
& \operatorname{vec}\left(-\boldsymbol{\theta}_{l} \boldsymbol{\theta}^{T}-\boldsymbol{\theta}_{l}^{T}+\boldsymbol{\theta}_{l} \boldsymbol{\theta}_{l}^{T}\right) \succeq \operatorname{vec}(-\boldsymbol{\Theta}) \\
& \operatorname{vec}\left(\boldsymbol{\theta}_{u} \boldsymbol{\theta}^{T}-\boldsymbol{\theta}_{u} \boldsymbol{\theta}_{l}^{T}+\boldsymbol{\theta}_{l}^{T}\right) \succeq \operatorname{vec}(\boldsymbol{\Theta})
\end{array}
$$

Results from our computer simulations show that the constraint of $\boldsymbol{\Theta} \succeq \boldsymbol{\theta} \boldsymbol{\theta}^{T}$ is tight, that is, the solution provided by (16) is identical to that of (10)-(12) when the noise power is small enough such that (3) holds. Note that in practice, we will employ $\mathbf{B}=\operatorname{diag}\left(d_{21}+\hat{r}, d_{31}+\hat{r}, \cdots, d_{M 1}+\hat{r}\right)$ to compute W where $\hat{r}$ is an estimate of $r$ which is obtained by minimizing (6) with $\mathbf{W}=\mathbf{I}_{4}$. After getting a more accurate $\hat{r}$ from (16), we can update $\mathbf{W}$ and then iterate the SDP algorithm again to attain better 
estimation performance. Regarding the computational requirement, the major arithmetic operations of the SDP approach come from the positive semi-definite constraint in (16) and the complexity is at least $O\left(m^{3}\right)$ [12] where $m=5$ in our case.

\section{Simulation Results}

Computation simulation has been performed to evaluate the positioning performance of the proposed SDP algorithm by comparing with the two-step WLS technique [6] and CRLB. We utilize the MATLAB toolbox YALMIP [9] to realize the SDP method where the solver SDPT3 [10]-[11] is employed. We consider the scenario of speaker localization in a room where the room dimension and speaker height range, $6 \mathrm{~m} \times 4 \mathrm{~m} \times 2 \mathrm{~m}$ and $1 \mathrm{~m} \leq z \leq 2 \mathrm{~m}$, which correspond to $\mathscr{P}$ and $\mathscr{S}$, respectively, are utilized as available prior information. It is assumed that there are eight microphones with positions $(0,0,0) \mathrm{m}$, $(6,0,0) \mathrm{m},(0,4,0) \mathrm{m},(6,4,0) \mathrm{m},(0,0,2) \mathrm{m},(6,0,2) \mathrm{m},(0,4,2) \mathrm{m}$ and $(6,4,2) \mathrm{m}$. The range errors are generated using $n_{i 1}=n_{i}-n_{1}$ where all $\left\{n_{i}\right\}$ are zero-mean white Gaussian processes with identical variance $\sigma^{2}$ [6]. The performance measure of mean square position error (MSPE) is considered and it is defined as $\mathbb{E}\left\{(\mathbf{x}-\hat{\mathbf{x}})^{T}(\mathbf{x}-\hat{\mathbf{x}})\right\}$ where $\hat{\mathbf{x}}$ denotes the estimate of $\mathbf{x}$. All results are averages of 500 independent runs.

In first experiment, the speaker is located at $\mathbf{x}=[3.5,2.5,1.5]^{T} \mathrm{~m}$, which corresponds to the central area of the room, and Figure 1 shows the MSPE performance versus $\sigma^{2}$ in dB scale. Three variants of the proposed approach, with no iteration and with 1 and 2 iterations, are investigated. It is observed that the SDP approach is superior to the two-step WLS method for larger noise conditions, that is, $\sigma^{2} \geq-45 \mathrm{dBm}^{2}$, and is able to attain the CRLB for nearly the whole range of $\sigma^{2}$. Furthermore, the estimation performance of the SDP algorithm with one and two iterations can give a higher accuracy when $\sigma^{2}>-30 \mathrm{dBm}^{2}$, which implies that one iteration is sufficient for optimality and no further iteration is required. As a result, we only show the results of no iteration and one iteration in the following. We also see that the MSE of the proposed approach is smaller than CRLB when $\sigma^{2} \geq-5 \mathrm{dBm}^{2}$. As the CRLB is a lower bound on the variance of any unbiased estimator, the only explanation for this phenomenon is that the SDP method becomes a biased estimator for larger noise conditions due to the constraints constructed 
from the a priori position knowledge. The corresponding mean absolute position error, which is defined as $\mathbb{E}\left\{\|\mathbf{x}-\hat{\mathbf{x}}\|_{1}\right\}$, is plotted in Figure 2 to demonstrate the biasedness of the the proposed scheme when the noise power is sufficiently large.

In second experiment, the speaker position is near to an edge of the room, namely, $\mathbf{x}=$ $[5.5,3.5,1.5]^{T} \mathrm{~m}$, and the MSPE performance versus $\sigma^{2}$ is shown in Figure 3. The superiority of the SDP approach over the 2-step WLS technique is again demonstrated, although the improvement is less significant compared with Figure 1. It is also seen that the iterative SDP algorithm gives no obvious gain over the standard one. Based on the numerical results, the small noise condition which makes (3) valid is around $\sigma^{2} \leq-30 \mathrm{dBm}^{2}$.

The computational complexity of the SDP approach in the first test has been studied. Based on a PC computer with Inter(R) Core(TM)2 CPU 6300@1.86GHz and 2 GB memory, the average CPU times for the SDP method with no iteration and two-step WLS technique are measured as $9.69 \times 10^{-4} \mathrm{~S}$ and $5.91 \times 10^{-2} \mathrm{~s}$, respectively, at $\sigma^{2}=-60 \mathrm{dBm}^{2}$. Although the latter involves a larger computational requirement, it is worthy to note that a tailor-made interior point method should reduce the complexity.

\section{CONCLUSION}

A convex optimization technique, namely, semi-definite programming (SDP) relaxation, has been derived for speaker localization using time delay measurements as well as the a priori position bounds deduced from the physical environment. The approximate optimality of the proposed positioning algorithm is demonstrated via computer simulations. It is shown that the SDP scheme outperforms the conventional two-step weighted least squares method in terms of threshold performance at the expense of a higher computational requirement. Furthermore, it becomes a biased estimator for larger noise conditions due to the constraints constructed from the prior position knowledge.

$\underline{\text { Acknowledgment }}$

The work described in this paper was supported by a grant from CityU (Project No. 7002132). 


\section{REFERENCES}

[1] H. Werstiuk, and A.E. Winter, The search and rescue satellite (SARSAT) system project, Proceedings of AGARD Symp. Operational Modeling of the Aerospace Environment, Ottawa, Canada, Apr. 1978.

[2] S.L.Gay and J.Benesty, Eds, Acoustic Signal Processing for Telecommunication, Norwell, MA: Kluwer Academic, 2000.

[3] J.J. Caffery, Wireless Location in CDMA Cellular Radio Systems, Kluwer Academic, 1999.

[4] J.B. Tsui, Fundamentals of Global Positioning System Receivers, John Wiley \& Sons, 2000.

[5] D.J.Torrieri, "Statistical theory of passive location systems," IEEE Trans. on Aerospace and Electronic Systems, vol.20, pp.183197, Mar. 1984.

[6] Y.T. Chan and K.C. Ho, "A simple and efficient estimator for hyperbolic location," IEEE Trans. Signal Processing, vol.42, pp.1905-1915, Aug. 1994.

[7] S. Boyd and L. Vandenberghe, Convex Optimization, Cambridge University Press, 2004.

[8] W.R. Hahn and S.A. Tretter, "Optimum processing for delay-vector estimation in passive signal arrays," IEEE Trans. Inform. Theory, vol.19, pp.608-614, Sep. 1973.

[9] J. Löfberg, "YALMIP : a toolbox for modeling and optimization in MATLAB," Proc. Int. Symp. CACSD, pp.284-289, Taipei, Taiwan, Sep. 2004.

[10] K.C. Toh, M.J. Todd and R.H. Tutuncu, "SDPT3 - a MATLAB software package for semidefinite programming," Optimization Methods and Software, vol.11, pp.545-581, 1999.

[11] R.H. Tutuncu, K.C. Toh and M.J. Todd, "Solving semidefinite-quadratic-linear programs using SDPT3," Mathematical Programming Ser. B, vol.95, pp.189-217, 2003.

[12] Z. Wang, S. Zheng, S. Boyd and Y. Ye, "Further relaxation of the SDP approach to sensor network localization," SIAM J. Optimization, vol.19, no.2, pp.655-673, Jul. 2008. 


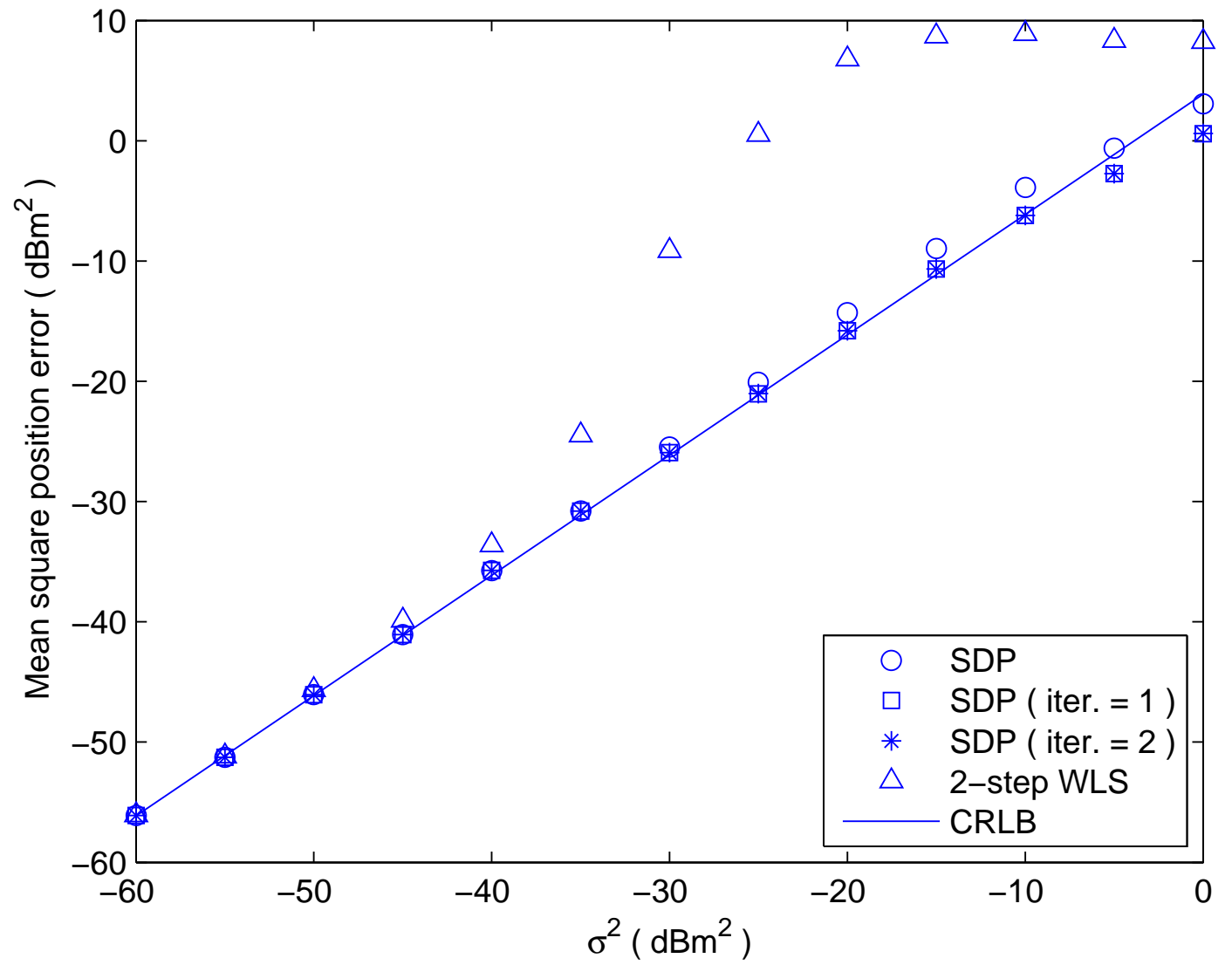

Fig. 1. Mean square position error versus $\sigma^{2}$ at $\mathbf{x}=[3.5,2.5,1.5]^{T} \mathrm{~m}$ 


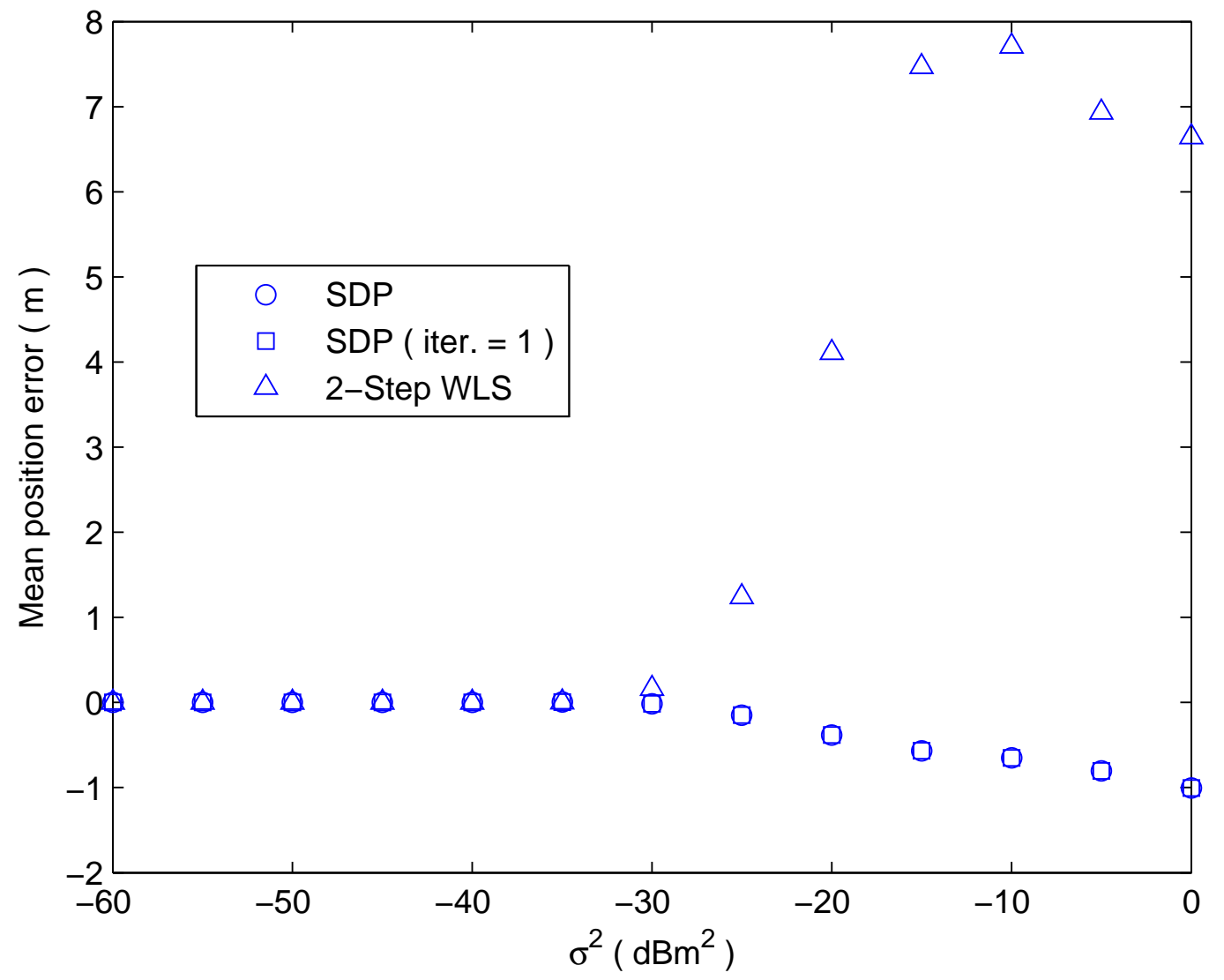

Fig. 2. Mean absolute position error versus $\sigma^{2}$ at $\mathbf{x}=[3.5,2.5,1.5]^{T} \mathrm{~m}$ 


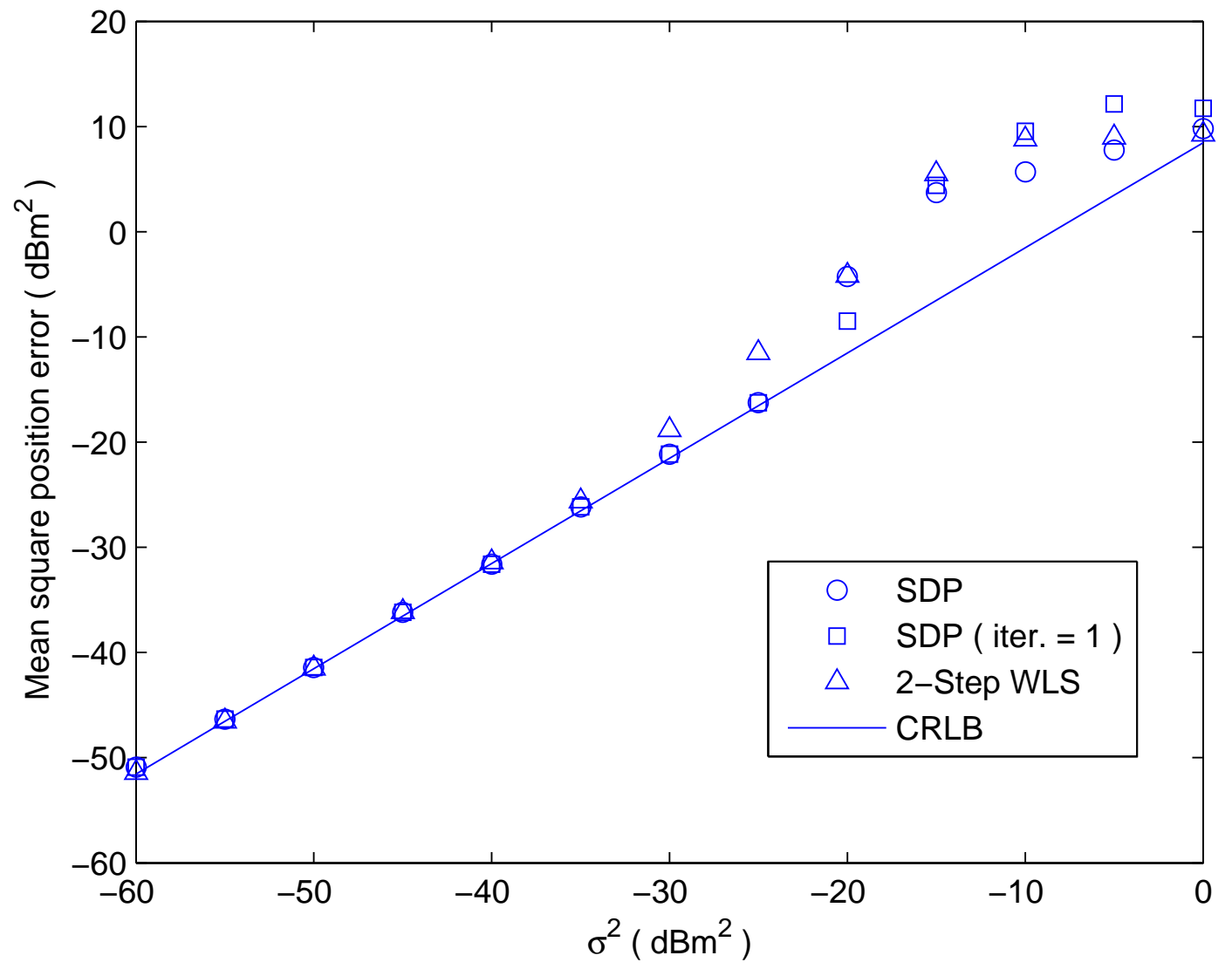

Fig. 3. Mean square position error versus $\sigma^{2}$ at $\mathbf{x}=[5.5,3.5,1.5]^{T} \mathrm{~m}$ 